\title{
In situ chip-based heating studies of metal-induced layer exchange and Si crystallization using STEM, LEND and SE imaging in SEM
}

Peter Denninger ${ }^{1}$, Peter Schweizer ${ }^{2}$, Tim Schwope ${ }^{1}$, Christian Dolle $^{3}$ and Erdmann Spiecker ${ }^{1}$

${ }^{1}$ Institute of Micro- and Nanostructure Research (IMN) \& Center for Nanoanalysis and Electron Microscopy (CENEM), Interdisciplinary Center for Nanostructured Films (IZNF), Department of Materials Science and Engineering, Friedrich-Alexander University Erlangen-Nürnberg (FAU), Erlangen, Germany, Erlangen, Bayern, Germany, ${ }^{2}$ Empa, Thun, Switzerland, ${ }^{3}$ Karlsruher Institut für Technologie, Karlsruhe, BadenWurttemberg, Germany

An indispensable tool for the analysis of phase transitions and material transport at high temperatures are in situ experiments. In material science a range of microscopes can be used for in situ experiments. Scanning electron microscopes (SEM) offer great potential for in situ investigations due to their low acceleration voltages, large chamber size and field of view, and strong topographic contrast. However, combining in situ heating with transmission imaging is a novel approach.

In this work, we introduce an update of our low-energy nanodiffraction (LEND) setup [1], which enables combined in situ heating, imaging, and transmission diffraction in SEM. Figure 1 illustrates the setup and shows exemplary LEND patterns of graphene and gold at different acceleration voltages. Implementing a custom-built heating stage for DENSsolutions heating chips (originally designed for TEM) allows precise and fast local heating and cooling of specimens. The setup has been implemented and successfully tested in solidstate dewetting experiments, where the simultaneous acquisition of real- and reciprocal space information in situ is needed to elucidate phenomena such as grain coarsening and texture evolution.

To showcase the benefits of combining several imaging modes in situ, we present current results about the crystallisation of thin amorphous Si layers via metal induced layer exchange (MILE). This process was intensively studied in the last two decades. A stack of metal, some native oxide, and amorphous Si inverts its stacking order while the Si crystallises (Figure 2a). Since the phenomenon of metal induced crystallization already occurs at temperatures much below the bulk crystallisation temperature of Si, the MILE process was investigated for its applicability in the fabrication of thin crystalline semiconductor films even on heat-sensitive substrates.

Annealing parameters for the $\mathrm{Ag} / \mathrm{Si}$ system were previously determined with respect to suitable reaction rates and morphology by in situ light microscopy experiments (Figure 2b). Characteristic dendritic growth of the individual $\mathrm{Si}$ grains can be observed, with the size and number of grains exhibiting strong temperature dependency. The overall sample thickness is also important for obtaining sufficient signal of the transmitted electrons.

During in situ heating experiments, all available signals were acquired to provide essential information about the phase transitions, crystal growth and orientation, change of grain structure, and topography. The phase transition from amorphous to crystalline caused the formation of a typical polycrystalline Si diffraction pattern, while crystal growth and layer exchange are observed through SE \& STEM imaging. High Angle Annular Dark Field (HAADF) Z-contrast imaging facilitates the distinction between Ag and Si due to their pronounced atomic number $(Z)$ difference. Figure 2c illustrates the observed Si crystallization during heating. By combining diffraction and imaging modes, the grain structure and orientation of the reacted area can be examined (Figure 2d). While the experimental results largely confirmed the most recent layer exchange model [2], we also observe differences based on which we propose a refined model for the AgILE process. 
In the future, simultaneous in situ diffraction and STEM imaging is planned, which expands the versatility of correlative in situ methods in the SEM. Additionally, EBSD/TKD measurements will be performed to further investigate grain orientation and morphology [3].

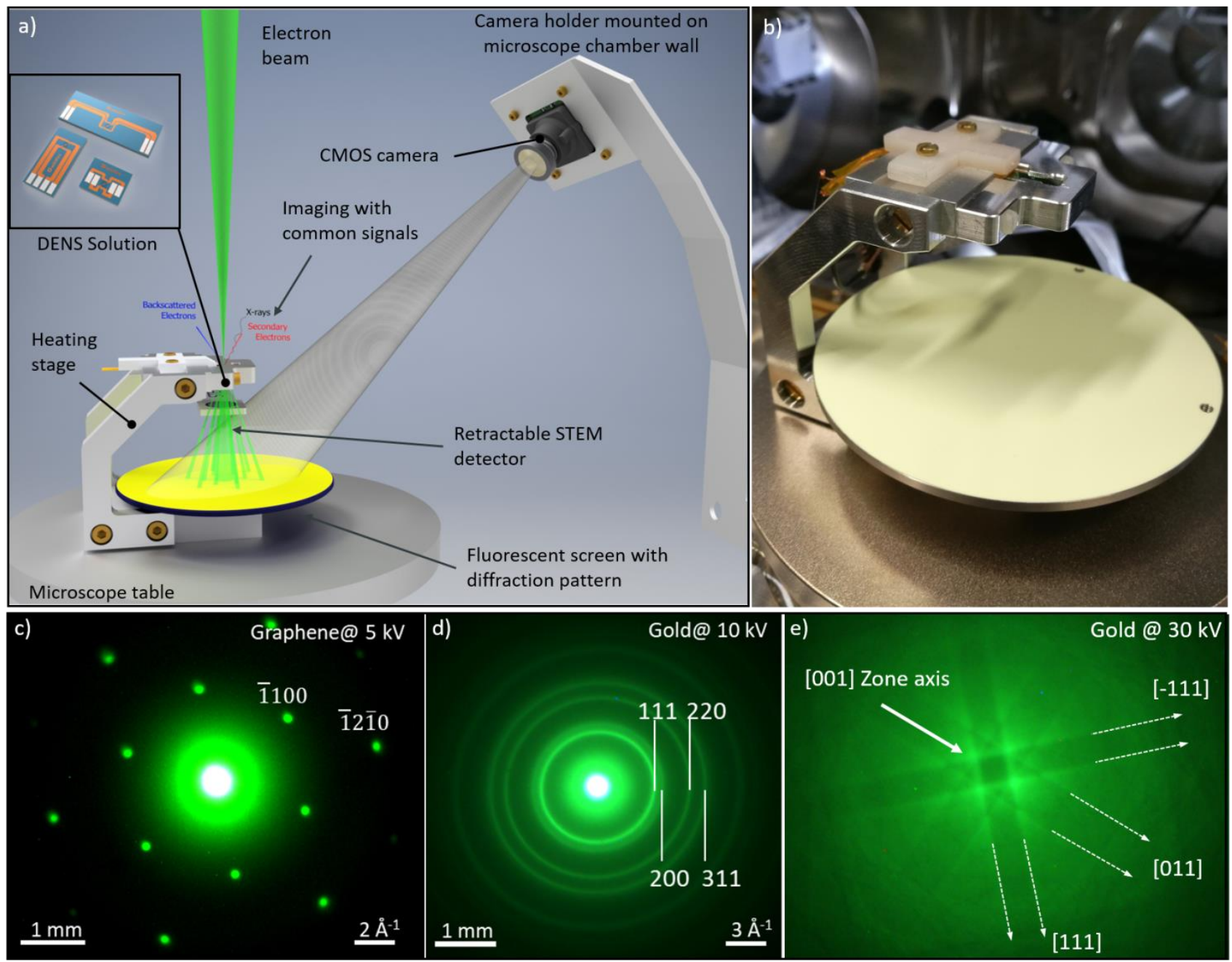

Figure 1. Figure 1: Combined in situ heating and low energy nanodiffraction (LEND) setup in the SEM. a) Setup of the custom-built heating stage for DENSsolutions chips. b) Installed heating stage in the SEM. c,d) Diffraction patterns of graphene and a polycrystalline gold film. e) Kikuchi diffraction for thicker material systems. 


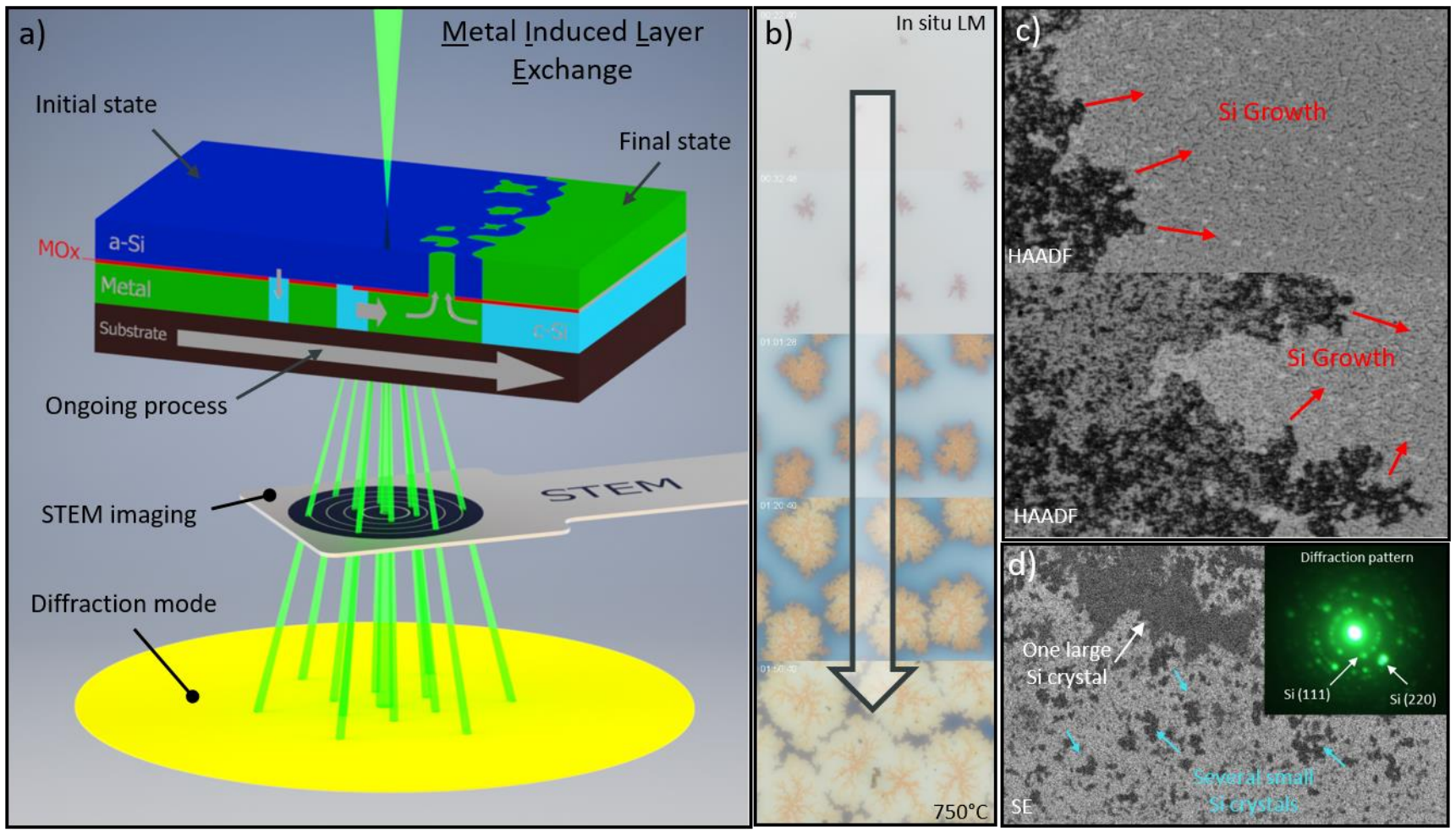

Figure 2. Figure 2: Investigation of the Metal Induced Layer Exchange system. a) Schematic of the MILEprocess. b) Pre-characterization of $\mathrm{Ag} / \mathrm{Si}$ system by in situ light microscopy. c) Crystallization of Si acquired by HAADF imaging. d) Overview of reacted area (SE) with corresponding diffraction pattern, which reveals grain structure and orientation.

\section{References}

[1] P. Schweizer, P. Denninger, C. Dolle, E. Spiecker, Low energy nano diffraction (LEND) - A versatile diffraction technique in SEM, Ultramicroscopy 213 (2020) 112956

[2] Z. Wang, L.P.H. Jeurgens, E.J. Mittemeijer (Eds.), Metal-induced crystallization: Fundamentals and applications, Pan Stanford Publishing, [Singapore], 2015

[3] The authors acknowledge funding by the German Research Foundation (DFG) via the Research Training Group GRK 1896 "In situ microscopy with electrons, X-rays and Scanning probes” 